\title{
Life events, Anxiety and Depression Among Doctors and Nurses in the Emergency Department : A Study From Eleven General Hospital in Hunan Province, China
}

JOURNAL OF PSYCHIATRY

AND BRAIN SCIENCE

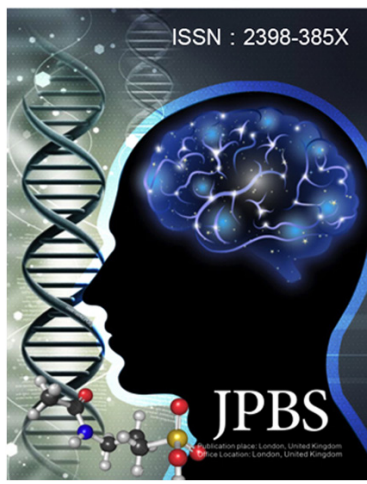

\section{GOPEN ACCESS}

DOI: 10.20900/jpbs.20160002

Received: January 13, 2016

Accepted: February 10, 2016

Published: April 25, 2016

website: http://jpbs.qingres.com

Copyright: (C2016 Cain et al. This is an open access article distributed under the terms of the Creative Commons Attribution License, which permits unrestricted use, distribution, and reproduction in any medium, provided the original author and source are credited.

Data Availability Statement: All relevant data are within the paper and its Supporting Information files.

Funding: The authors received no specific funding for this work.

Competing Interests: The authors have declared that no competing interests exist.

\begin{abstract}
Yamin $\mathrm{Li}^{1}$, Hongliang Zhang ${ }^{1}$, Zhijuan Feng ${ }^{2}$, Shubao Chen ${ }^{2}$, Tieqiao $\mathrm{Liu}^{2^{*}}$; Xiaogang Chen ${ }^{2}$; Jinsong Tang ${ }^{2^{*}}$; Yanhui Liao ${ }^{2^{*}}$

1. Department of Emergency, the Second Xiangya Hospital of Central South Universily, 139 Renmin (M) Rd, Changsha, Hunan, 410011, P.R.China.

2. Mental Health Institute of the Second Xiangya Hospital, Central South University, The China National Clinical Research Center for Mental Health Disorders, National Technology Institute of Psychiatry, Key Laboratory of Psychiatry and Mental Health of Hunan Province, 139 Renmin (M) Rd, Changsha, Hunan, 410011, P.R.China.

*Corresponding: Dr. Yanhui Liao, email: tangliaoyanhui@gmail.com, Dr. Jinsong Tang, email: tangjinsonghn@gmail.com, 139 Renmin (M) Rd, Changsha, Hunan, 410011, P.R.China.
\end{abstract}

\section{ABSTRACT}

Objectives:To evaluate life events, levels of anxiety and depression among doctors and nurses in the emergency department (ED).

Design:An anonymous self-reported survey was employed from January 2013 to April 2013 that included Life Event Scale (LES), Zung's Self-Rating Anxiety Scale (SAS), Zung's Self-Rating Depression Scale (SDS) and other Likert-style questions.

Participants:A total sample of 412 participants (124 doctors and 288 nurses) from department of emergency in eleven grade-3 and grade-2 general hospitals in Hunan Province, China was involved in this study.

Results:Both doctors and nurses from ED experienced more negative life events than positive life events (mean total score: 14.13 VS. 6.41). Among all family, work and social life events, high pressures of work as negative life event was the most frequently reported one. $6.34 \%$ (5.65\% in doctors and $6.60 \%$ in nurses) suggestive anxiety symptoms and $17.73 \%$ (14.5\% in doctors and $18.8 \%$ in nurses) suggestive depression symptoms were reported among them. Negative, but not positive, life events correlated with symptoms of depression and anxiety. Doctors experienced much more work related negative events than nurses did. However, nurses showed higher levels of anxiety and depression that that of doctors.

Conclusion:These findings suggest doctors and nurses from emergency department experienced much more negative life events than positive life events, and those negative life events, especially work related negative events, may have harmful impact on their mental health (risk for depression and anxiety). Development of a range of coping strategies for decreasing the harmful effects of those negative life events and minimizing or controlling the occurrence of anxiety and depressive symptoms should be emphasized for doctors and nurses 
who work in a hospital's emergency department.

Key words: life events; depression; anxiety; emergency department; doctors; nurses; Chinese

\section{INTRODUCTION}

The term stress, which have been introduced into the health sciences in 1926 by Hans Selye, refers to a state characterized by a uniform response pattern, regardless of the particular stressor, that could lead to long-term pathologic changes [1]. The chronic stressors, such as environmental and psychosocial stressors, are associated with physiological, psychological, and behavioral responses. Stress has often been conceptualized as major life events that are disrupting normal activities and often requiring adaptive responses, and as daily life events (i.e. chronic stressors) that are defined as the problems, difficulties, and challenges that people experience during extended periods of time in their daily lives [2]. For example, divorce could be recognized as a major life event and unsatisfied with the job can be considered as a chronic stressor. Markers of daily life stress reactivity may increase the risk of mental disorders [3].

Depression [4] and anxiety [5] are one of the most prevalent and comorbid psychiatric illnesses. Depression and anxiety have been associated with low levels of physical function [6]. Depression is one of the most important burden of diseases [7] and causes of global disability-adjusted life year [8]. The National Co-Morbidity Replication Survey conducted between February 2001 and April 2003 estimated that the lifetime prevalence of major depression to be $16.9 \%$ overall with a higher prevalence in females $(20.2 \%)$ compared with males $(13.2 \%)$, the lifetime prevalence of anxiety disorders was $28.8 \%$ [9]. Stressful life events have been associated with the onset of generalized anxiety disorder [10] and number of episodes and depression severity [11]. Spinhoven et al found that specific adverse life events, both in early life and adulthood were associated with anxiety and depressive disorders, and with a higher comorbidity of affective disorders [12].

Emergency department is recognized as a stressful environment that often exerts a negative effect on the physical health and psychosocial well-being of the doctors and nurses. A line of researches have indicated a direct relationship between stress and illness in general [13]. It has been generally accepted that some major life events, such as broken marriage and death of family members or friends, are associated with physical and mental health impairments in individuals. The underlying assumption is that such events serve as precipitating factors, influencing the timing but not the type of illness episodes [14]. Studies found that work-related, personal and family-related stressful life events contribute to the development and/or course of chronic diseases (Renzaho, Houng et al. 2013), and that stressful life events have been associated with the onset of generalized anxiety disorder [10] and number of episodes and depression severity [15]. Although there is a growing concern about mental health in general population [16]. There is a limitation in the research of life events and levels of depression and anxiety in doctors and nurses from emergency department whom have been daily exposed to intensive and stressful working environment. What is less clear, however, is whether accumulating daily life events are presenting sustained impact on them.

The purpose of this study was to assess the timing and number of specific experienced life events (including family and marriage, work and study, social and others life events) and to examine the impact of those life events on doctors and nurses whom are working in emergency department where often considered as stressful working environment. Given that doctors and nurses from emergency department are working under high stress, we hypothesized that negative life events from work (rather that family and social) would be the most frequently reported ones. Also, given that the timing and number of stressors are contributing to the risk for of depression and anxiety, we also hypothesized that high levels of life events are associated with negative outcomes, such as depression and anxiety.

\section{METHODS}

\section{Design}

A cross sectional descriptive self-reported survey design was used to explore life events, levels of anxiety and depression in a sample of doctors and nurses from department of emergency.

\section{Participants}

The present study employed a self-report survey design. Entry into the study was between January 2013 and April 2013. A total sample of 412 participants (124 doctors and 288 nurses) from department of emergency in eleven grade-3 and grade-2 general hospitals in Hunan Province of China was involved in this study. Apart from working in the department of emergency as a doctor or nurse, there were no exclusion 
criteria. Participation was voluntary and they could withdraw at any time of the study without prejudice or consequence to their work.

\section{Assessment Measures}

In addition to some demographic and functional variables, the following self-administered questionnaires were used: Life Event Scale (LES), Zung's Self-Rating Anxiety Scale (SAS) and Zung's Self-Rating Depression Scale (SDS). Fatigue Assessment Instrument (FAI) and Pittsburgh Sleep Quality Index (PSQI) were also included in the battery of measures. Details of FAI and PSQI will be discussed separately. All participants were invited to complete a battery of self-administered measures.

1. Life Event Scale (LES) [17]: the LES is a 48-item self-report measure designed to assess positive and negative life events. It includes 28 items of family and marriage related events, 13 items of work and study related events and 7 items of social related and other events. It has been widely used in assessing life events experienced by individuals with psychosocial stress and illness as well as general population in China. The participants were asked to rate which of the list of life events had occurred. And then, they were required to classify when it happened (score for 0 to 3 points: never happened, within the previous 6 months, more than 6 months and long-term), to definite the occurred event as positive or negative event, to rate the degree of its mental effect (score for 0 to 4 points: not at all, mild, moderate, severe and extremely severe), and to classify the duration of this mental effect (score for 1 to 4 points: within 3 months, within 6 months, within 1 year and more than 1 year). Total scores of each happened event $=$ scores of when it happened $\times$ scores of degree of its mental effect $\times$ scores of the duration of this mental effect; total negative scores $=$ added scores of each negative event; total positive scores= added scores of each negative event. High negative scores could mean high stress, anxiety, distress and so on. LES is a Chinese version of questionnaire and widely used in China with high level of reliability and validity [17], and it was used for heroin users in our previous study [18].

2. Zung's Self-Rating Anxiety Scale (SAS) [19,20]: the SAS is a 20-item (e.g.,' I feel more nervous and anxious than usual.'), self-reported rating scale designed to assess anxiety. Items are scored on a likert-scale ranging from 0 (not at all) to 4 (extremely) with scores ranging between 0 and 80 . Higher scores indicate higher levels of anxiety.

3. Zung's Self-Rating Depression Scale (SDS) [21,22]: the SDS is a 20-item questionnaire assessing mood symptoms over the past week (e.g., 'I feel downhearted, blue and sad'). Each item is scored on a likert-scale ranging from 1 (never) to 4 (always). Higher scores indicate higher levels of depression.

\section{Procedure}

Prior to the survey, the protocol was approved by the university ethics committee (The Second Xiangya Hospital of Central South University Review Board, No. S101, 2011) and the studies were carried out in accordance with the Declaration of Helsinki. All clinical workers from those eleven general hospitals of emergency department in Changsha city were invited to participate in the study during the free time. It was emphasized that participation in this study was completely voluntary and anonymously, and that participants were free to withdraw at any time. Participants were encouraged to answer all the questions independently within one hour. Participants were fully informed about the measurement in the study. Oral informed consent was given by all study participants.

\section{Statistical Analyses}

Statistical analysis was performed using the SPSS for Windows (Version 22, SPSS Inc., Chicago, IL, USA) software package. To address missing data, 4 incomplete questionnaires were excluded. Descriptive statistics were used to explore the study population's characteristics and pooled responses. Independent sample T-tests and Pearson's chi-square test were used to determine differences between doctors and nurses in terms of life events, anxiety and depression. Correlations between life events and symptoms of anxiety and depression were explored using the Pearson Correlation. An alpha level of 0.05 was set to determine statistical significance.

\section{RESULTS}

\section{Sample Characteristics}

Among 450 invited participants, 408 participants (90.67\% response rate) returned completed questionnaire including 124 doctors (30.39\%) and 284 nurses (69.61\%). 3. Detailed socio-demographic characteristics see table 1. Nationality: 383 (93.0\%) Han Chinese, 29 (7.0\%) minorities. Marital status: 225 (54.6\%) married, 187 (45.4\%) unmarried. Educational level: 352 (85.4\%) Nursing school or college, 60 (14.6\%) above college. 


\section{The Frequency of Life Events}

Detailed life events reported by 408 participants have been shown in table 2 and table 3 . According to LES response, the most frequently reported (17.40\%, 71 participants) life events was the experiences of high pressures of work that have negative impact on them. Falling in love or engagement, getting married, to start the employment, gaining a new family member, I am (or my wife is) pregnancy, getting a promotion and significant economic improvement are the frequently reported positive life events; under high pressures of work, unsatisfied with current job, significant lifestyle pattern changes (dietary and sleep), withholding bonus or getting penalty, failure in love affair, being separated from my spouse (work required), be in debt, housing shortage, be misunderstood, unjustly blamed, falsely charged and discussed, conflict with parents-in-law, family financial problems, significant economic improvement, poor relationship in spouse, family member(s) has/have serious illness and/or serious injury are the frequently reported negative life events. In terms of the first time in foreign country or at alien place, four participants reported it as positive life events while one subject reported it as negative life events (see table 2).

Compare with total scores of positive life events, participants reported higher scores of negative life events, and doctors experienced more positive and negative life events than nurses (see table 3).

\section{Symptoms of Anxiety and Depression}

High aggregate mean scores were observed suggesting high levels of anxiety. 26 (6.37\%, 6 males doctors and 20 females with 1 doctor and 19 nurses) participants obtained SAS scores $\geq 50$ and 72 (17.65\%, 15 males with 13 doctors and 2 nurses and 57 females with 5 doctors and 52 nurses) participants obtained SDS scores $\geq 50$ which are indicative high anxiety and depression symptoms respectively in these participants (see table 4$)$. To specific, $3(0.73 \%)$ participants obtained scores $\geq 60,23(5.61 \%)$ participants obtained scores between $<60$ and $\geq 50$, and $31(7.56 \%)$ participants obtained scores between $<50$ and $\geq 45$ on the SAS which are indicative of severe, moderate and mild anxiety respectively. The same goes for SDS, high aggregate mean scores were observed suggesting high levels of depression. $7(1.72 \%)$ participants obtained SDS scores in the very upper ranges ( $\geq 60$ scores) indicating very high level of depression symptoms while 65 $(16.01 \%)$ participants obtained scores between $<60$ and $\geq 50$ and $57(14.04 \%)$ participants obtained scores between $<50$ and $\geq 45$ on the SDS which are indicative higher depression symptoms in these participants. No significant gender and occupation (nurses and doctors) differences were found on the SDS ( $p=0.751$, $p=0.720)$ and one the SAS $(p=0.577, p=0.331)$.

\section{Correlations Between Life Events and Symptoms of Anxiety and Depression}

Pearson correlation analyses were conducted between total positive score, total negative score and SAS (anxiety), SDS (depression). In terms of total negative LES score, it was correlated with both anxiety ( $r p=0.311$, $\mathrm{p}<0.000)$ and depression $(\mathrm{r} p=0.200, \mathrm{p}<0.000)$, while total positive LES score was not correlated with anxiety $(r p=0.039, p=0.427)$ and $(r p=-0.041, p=0.406)$.

\section{DISCUSSION}

To our knowledge, this pilot study is the first to investigate the detailed family and marriage, work and study, social and others life events, and its association with symptoms of anxiety and depression among doctors and nurses from department of emergency in Hunan Province, China.

First of all, we found that the frequently reported positive life events were falling in love or engagement, getting married, to start the employment, gaining a new family member and negative life events were under high pressures of work, unsatisfied with current job, significant lifestyle pattern changes (dietary and sleep) (see table 2). Furthermore, participants reported more negative life events (14.1) than positive life events (6.4) and doctors experienced more negative and positive life events than nurses did (see table 3 ). It is worth pointing out that $17.40 \%$ (71 participants) reported high pressures of work as negative impact, which was the most frequently reported life events (see table 2) and consistent with our first hypothesis. In China, health care staffs are often suffering from heavily physical and psychological burdens, especially those emergency health care providers [23]. Besides the pressures from clinical work, Chinese doctors often have to face the pressure of publication, which is one the critical requirements for getting promotion [24]. This may partly explain why doctors experienced more negative life events than nurses. Why doctors experienced more negative and positive life events than nurses? Another possible explanation is that doctors and nurses have experience difference levels and sources of stress and their show different cope strategies for stress. For example, Goodfellow et al. found that nursing staff have different sources of stress from doctors and individuals with partners or children are relatively protected from stress [25]. As for working related life events, doctors may exact greater commitment to their work when compared with nurses [26], which may 
expose doctors under higher working pressures.

Secondly, the study revealed that suggestive anxiety symptoms were in $6.37 \%$ participants and suggestive depression symptoms were in $17.65 \%$ participants (see table 4 ). Although the estimated prevalence of anxiety and depression found in this study would not be higher than that in general population $[5,27]$, we have to pay close attention to those symptoms as self-treatment for mental illness is common in medical workers. It is reported that physicians do not seek the kind of professional help for themselves as they do for their patients [28], especially when they were suffering from mental health problems, most of them would seek help for mental problems elsewhere than in the mental health care facilities located where they lived [29]. Destigmatization of mental problems in physicians and interventions to improve the mental health care of physicians in ways that do not compromise their professional standing should receive more attention.

In addition, there were no statistical significances between nurses and doctors in terms of the prevalence of anxiety and depression (see table 4). However, the mean of SDS total scores in current study was slightly higher than a university medical students sample from Changsha (39.0 VS. 37.4), and the mean of SAS total scores was strikingly higher in this sample than the medical students sample (36.0 VS. 24.8) [30], which indicated that medical staffs from emergency department exhibit high levels of anxiety when compare with medical students.

We also found that nurses had higher total mean score of SAS (anxiety) and SDS (depression) than doctors (see table 4 ). As the majority $(94.37 \%)$ of nurses and only one third $(29.32 \%)$ of doctors were female in this study, we speculated that these differences may due to the gender differences of depression and anxiety [31].

Finally, we explored the correlation between total positive score, total negative score and SAS (anxiety), SDS (depression). Total negative LES score was correlated with both anxiety and depression, while total positive LES score was neither correlated with anxiety nor depression. These findings were consistent with our second hypothesis (life events associate with depression and anxiety) and results from previous studies. For example, Spinhoven et al. found that life events were significantly related to diagnostic and symptom course trajectories of depression and anxiety, and only negative life events have an independent effect on diagnostic and symptom course trajectories of depression [32]; Fox et al. found that negative life event linked to greater depressive symptoms [33]. The current study showed no association between positive life events and depression and anxiety, which does not concur with the conclusions of review of the literature on positive life events with depression and anxiety. Previous research indicated that positive life events would trigger recovery from depression and anxiety and predict better outcome in depressed patients [34,35]. The impacts of the positive life events on this sample and other general population require further investigation. Further research is also required to better understand the impact of negative life events on chronic disease in high stressed emergency department doctors and nurses.

\section{STRENGTHS AND LIMITATIONS}

A significant strength of this study is that we not only assessed the incidence of positive and negative life events refer to family and marriage, work and study, social and other aspects, and we did ask participants to rate the severity and duration of the impact of these life events. To the best of our knowledge, no previously study had explored positive and negative minor or major life events and their association with anxiety and depression in Chinese medical workers (doctors and nurses) from highly intensive working environment (emergency department). However, there are several limitations of this study. First, all data was collected using self-report measures which may have influenced the rates of at-risk depression and/or anxiety disorders observed. Studies using more costly and time-consuming interview-based methods will be superior to self-report methods in terms of detecting associations with depression and anxiety. Another weakness we should acknowledge is the fact that the results of our observations were likely biased because of the less extensive sample (408 participants). But it is noticeable that given the limitations of sample size and selectivity, the general yet specific life events were well measured. Finally, the sample was recruited only from department of emergency. Therefore it may be more difficult to generalize beyond those relatively highly stressed populations.

\section{CONCLUSION}

In conclusion, this study reported that doctors and nurses from emergency department experienced more 
negative life events than positive life events; the most frequently reported life event was high pressures of work; only negative (not positive) life events correlated with symptoms of depression and anxiety; $6.37 \%$ suggestive anxiety symptoms and $17.65 \%$ suggestive depression symptoms among them. Since having a repertoire of strategies to employ leads to greater effectiveness than simply relying on one strategy [36], it suggests that development of a range of coping strategies for decreasing the harmful effects of those negative life events and minimizing or controlling the occurrence of anxiety and depressive symptoms should be emphasized for those from emergency department and other population. For example, psychological interventions such as cognitive behaviour therapy or meditation-based techniques may be indicated, as well as health education interventions and policy strategies could be applied for those emergency department doctors and nurses.

\section{Acknowledgements}

This work was supported by grants from the Specialized Research Fund for the Doctoral Program of Higher Education (20110162120013) and Hunan Provincial Natural Science Foundation of China (12JJ3112). The grants had no further role in study design; in the collection, analysis and interpretation of data; in the writing of the report; or in the decision to submit the paper for publication.

\section{Author Contributors}

Authors Yanhui Liao and Jinsong Tang wrote the first draft of the manuscript. Author Yanhui Liao managed the literature searches. All authors contributed to and have approved the final manuscript.

\section{Declaration of Interest}

The authors report no conflicts of interest. The authors alone are responsible for the content and writing of the paper.

\section{REFERENCES}

1. Goldstein DS, Kopin IJ (2007) Evolution of concepts of stress. Stress 10: 109-120.

2. Israel BA, Farquhar SA, Schulz AJ, James SA, Parker EA (2002) The relationship between social support, stress, and health among women on Detroit's East Side. Health EducBehav 29: 342-360.

3. Collip D, Wigman JT, Myin-Germeys I, Jacobs N, Derom C, et al. (2013) From epidemiology to daily life: linking daily life stress reactivity to persistence of psychotic experiences in a longitudinal general population study. PLoSOne 8: e62688.

4. Luppa M, Sikorski C, Luck T, Ehreke L, Konnopka A, et al. (2012) Age- and gender-specific prevalence of depression in latest-life--systematic review and meta-analysis. J AffectDisord 136: 212-221.

5. Kessler RC, Ruscio AM, Shear K, Wittchen HU (2010) Epidemiology of anxiety disorders. CurrTopBehavNeurosci 2: 21-35.

6. Stegenga BT, Nazareth I, Torres-Gonzalez F, Xavier M, Svab I, et al. (2012) Depression, anxiety and physical function: exploring the strength of causality. J EpidemiolCommunity Health 66: e25.

7. Merry S, McDowell H, Hetrick S, Bir J, Muller N (2004) Psychological and/or educational interventions for the prevention of depression in children and adolescents. CochraneDatabaseSystRev: CD003380.

8. Murray CJ, Lopez AD (1997) Global mortality, disability, and the contribution of risk factors: Global Burden of Disease Study. Lancet 349: 1436-1442.

9. Kessler RC, Berglund P, Demler O, Jin R, Merikangas KR, et al. (2005) Lifetime prevalence and age-of-onset distributions of DSM-IV disorders in the National Comorbidity Survey Replication. ArchGenPsychiatry 62: 593-602.

10. Francis JL, Moitra E, Dyck I, Keller MB (2012) The impact of stressful life events on relapse of generalized anxiety disorder. DepressAnxiety 29: 386-391.

11. Roca M, Gili M, Garcia-Campayo J, Armengol S, Bauza N, et al. (2013) Stressful life events severity in patients with first and recurrent depressive episodes. Social psychiatry and psychiatric epidemiology 48: 1963-1969.

12. Spinhoven P, Elzinga BM, Hovens JG, Roelofs K, Zitman FG, et al. (2010) The specificity of childhood 
adversities and negative life events across the life span to anxiety and depressive disorders. $J$ AffectDisord 126: 103-112.

13. Rosch PJ (1979) Stress and illness. JAMA 242: 417-418.

14. Rabkin JG, Struening EL (1976) Live events, stress, and illness. Science 194: 1013-1020.

15. Renzaho AM, Houng B, Oldroyd J, Nicholson JM, D'Esposito F, et al. (2014) Stressful life events and the onset of chronic diseases among Australian adults: findings from a longitudinal survey. The European Journal of Public Health 24: 57-62.

16. Hardeveld F, Spijker J, De GR, Nolen WA, Beekman AT (2013) Recurrence of major depressive disorder and its predictors in the general population: results from The Netherlands Mental Health Survey and Incidence Study (NEMESIS). PsycholMed 43: 39-48.

17. Yang D, Zhang Y (1999) Life events scale. Chinese Mental Health Journal 12: 101-101.

18. Liao Y, Tang J, Liu T, Chen X, Liu X, et al. (2011) A pilot study of life events and mood disorders: selfreport survey in chinese heroin-dependent individuals. AmJ Addict 20: 337-342.

19. Zung WW (1971) A rating instrument for anxiety disorders. Psychosomatics 12: 371-379.

20. Wang Z, Chi Y (1984) Chinese version of Zung's self-rating anxiety scale. J Shanghai Psychiatry 2: 7374.

21. Shu L, Wang X, Wang X, Ma H (1999) A self-rating depression scale. Chinese Mental Health Journal 194: 196.

22. Zung WW, Richards CB, Short MJ (1965) Self-rating depression scale in an outpatient clinic: further validation of the SDS. Archives of general psychiatry 13: 508-515.

23. Huang W, Liu J, Yu X (2016) Sudden death: A growing threat to doctors in China. International Journal of Cardiology.

24. Yuan H, Xu W, Hu H (2013) Young Chinese doctors and the pressure of publication. The Lancet 381: e4.

25. Goodfellow A, Varnam R, Rees D, Shelly MP (1997) Staff stress on the intensive care unit: a comparison of doctors and nurses. Anaesthesia 52: 1037-1041.

26. Fogaça MdC, Carvalho WBd, Cítero VdA, Nogueira-Martins LA (2010) Preliminary study about occupational stress of physicians and nurses in pediatric and neonatal intensive care units: the balance between effort and reward. Revista latino-americana de enfermagem 18: 67-72.

27. Schwenk TL, Gorenflo DW, Leja LM (2008) A survey on the impact of being depressed on the professional status and mental health care of physicians. J ClinPsychiatry 69: 617-620.

28. Toyry S, Rasanen K, Kujala S, Aarimaa M, Juntunen J, et al. (2000) Self-reported health, illness, and self-care among finnish physicians: a national survey. ArchFamMed 9: 1079-1085.

29. Rosvold EO, Bjertness E (2002) Illness behaviour among Norwegian physicians. ScandJ Public Health 30: $125-132$.

30. Liao Y, Knoesen NP, Deng Y, Tang J, Castle DJ, et al. (2010) Body dysmorphic disorder, social anxiety and depressive symptoms in Chinese medical students. SocPsychiatry PsychiatrEpidemiol 45: 963-971.

31. Altemus M, Sarvaiya N, Epperson CN (2014) Sex differences in anxiety and depression clinical perspectives. Frontiers in neuroendocrinology 35: 320-330.

32. Spinhoven P, Elzinga BM, Hovens JG, Roelofs K, van OP, et al. (2011) Positive and negative life events and personality traits in predicting course of depression and anxiety. Acta PsychiatrScand 124: 462-473.

33. Fox JK, Halpern LF, Ryan JL, Lowe KA (2010) Stressful life events and the tripartite model: relations to anxiety and depression in adolescent females. J Adolesc 33: 43-54.

34. Leenstra AS, Ormel J, Giel R (1995) Positive life change and recovery from depression and anxiety. A three-stage longitudinal study of primary care attenders. BrJ Psychiatry 166: 333-343.

35. Paykel ES (2003) Life events and affective disorders. Acta PsychiatrScandSuppl: 61-66.

36. Phillips LJ, Francey SM, Edwards J, McMurray N (2009) Strategies used by psychotic individuals to cope with life stress and symptoms of illness: a systematic review. AnxietyStressCoping 22: 371-410. 